\title{
ÉVOLUTION DU DIVORCE PAR LA VOLONTÉ UNILATÉRALE HORS FAUTE DANS LA LÉGISLATION FRANÇAISE MODERNE: D’UN DIVORCE DUR À UN DIVORCE ASSOUPLI*
}

\author{
Evolución del divorcio por voluntad unilateral fuera de culpa en la legislación francesa \\ moderna: de un divorcio difícil a un divorcio más flexible
}

Afrah ALATI

\begin{abstract}
Sommaire:
Introducción I. Le modèle du divorce pour rupture de la vie commune: dureté dans son mécanisme, sanction par les effets A. Divorce dur dans son mécanisme B. Divorce sanctionné par les effets II. Le modèle du divorce pour altération définitive du lien conjugal: souplesse dans son mécanisme, absence de sanction par les effets A. Divorce plus souple dans son mécanisme B. Divorce non sanctionné par les effets III. Conclusion
\end{abstract}

Résumé: Le divorce par une seule volonté unilatérale d'un époux, sans s'appuyer sur une faute quelconque imputable au conjoint défendeur, a connu une évolution considérable dans la législation française moderne. L’expérience française en l'espèce a révélé deux modèles qui reflètent à quel point le législateur français a fait évoluer sa philosophie législative à l'égard de ce type de divorce. Dans un premier temps, le législateur de 1975 a intégré le divorce pour rupture de la vie commune. Ce type de divorce a été caractérisé par la dureté dans son mécanisme et la sanction par les effets. À côté des conditions strictes à réunir, le législateur, à l'époque, a en plus imposé une clause de dureté pour mettre un frein au divorce et éviter l'automaticité de son prononcé. De plus, la loi a en quelque sorte sanctionné le demandeur de ce divorce en mettant tous les effets du divorce à sa charge.

En revanche, trente ans après, le législateur de 2004 a présenté un autre modèle sous la forme $d u$ divorce pour altération définitive du lien conjugal. Dans ce modèle, le législateur a changé complètement son traitement législatif qui se caractérise par la souplesse dans le mécanisme de divorce et l'absence de sanction par les effets. Pour faciliter la demande de ce divorce, le législateur s'est concentré, d'une part, à atténuer les conditions exigées vis-à-vis de l'ancien divorce pour rupture de la vie commune et, d'autre part, sur la suppression de la clause de dureté. Plus encore, si l'ancien divorce pour rupture de la vie commune faisait peser systématiquement toutes les charges et conséquences du divorce sur le demandeur, la loi du 26 mai 2004 a choisi le contraire. Les effets du divorce, y compris celui pour altération définitive du lien conjugal, sont dorénavant soumis à un droit commun. En effet, ce divorce n'est plus un divorce assumé par une contrepartie.

\footnotetext{
* Cet article est offert à Mme Marcela Leticia López Serna, qui m’a exhorté à écrire dans le Journal juridique de l'Université de Guanajuato.

** Maître de conférences en droit privé à l'Université d'Asmarya- Libye. Doctorat en droit privé de l'Université d’Orléans-France. Diplômée d'études supérieures en droit islamique de l’Université de Tripoli - Libye
} 
En somme, on est passé d'une culpabilité systématique à un droit au divorce décomplexé pour le demandeur de ce divorce et d'un divorce pour cause objective restreinte à un divorce pour cause objective plus libérale.

Resumen : El divorcio por voluntad unilateral de un cónyuge, sin basarse en culpa alguna imputable al cónyuge demandado, ha experimentado una evolución considerable en la legislación francesa moderna. La experiencia francesa en este caso ha revelado dos modelos que reflejan hasta qué punto el legislador francés ha cambiado su filosofía legislativa con respecto a este tipo de divorcio. Como primer paso, el legislador de 1975 incluyó el divorcio para la ruptura de la vida común. Este tipo de divorcio se caracterizaba por la dureza de su mecanismo y la sanción por los efectos. Además de las estrictas condiciones que debían cumplirse, el legislador, en su momento, también impuso una cláusula de dureza para frenar el divorcio y evitar el carácter automático de su pronunciamiento. Además, la ley ha sancionado de alguna manera al demandante de este divorcio al poner a su cargo todos los efectos del divorcio.

Por otra parte, treinta años después, el legislador de 2004 presentó otro modelo en forma de divorcio para la alteración permanente del vínculo conyugal. En este modelo, el legislador ha cambiado completamente su tratamiento legislativo, que se caracteriza por la flexibilidad en el mecanismo de divorcio y la ausencia de sanciones por efectos. Para facilitar la solicitud de divorcio, el legislador se centró, por una parte, en la reducción de las condiciones requeridas en el anterior divorcio para la ruptura de la vida común y, por otra, en la abolición de la cláusula de dureza. Además, si el anterior divorcio por ruptura matrimonial imponía sistemáticamente todos los cargos y consecuencias del divorcio al demandante, la ley de 26 de mayo de 2004 optó por lo contrario. Los efectos del divorcio, incluido el de la alteración permanente del vínculo conyugal, están ahora sujetos al derecho consuetudinario. De hecho, este divorcio ya no es un divorcio asumido por una contraparte.

En resumen, hemos pasado de una culpabilidad sistemática a un derecho de divorcio libre de complejos para el demandante de este divorcio y de un divorcio por una razón objetiva limitada a un divorcio por una razón objetiva más liberal.

\section{Introduction}

Le divorce comme moyen de rompre le mariage présente, quant à son principe même, une histoire particulière en France. D’une manière générale, la législation du divorce, dans ce pays de culture catholique, a connu une suite de rebondissements ${ }^{1}$ qui a consacré tantôt son éclipse totale, tantôt sa reconnaissance avec des restrictions. Le divorce pour faute n'a été intégré, en France, qu'en $1884 .^{2}$ Pendant 91 ans, le législateur français a adopté la conception du divorce-sanction comme seule cause pour la rupture du mariage des époux vivants. Le grand point de revirement n’est venu que dans le dernier quart du siècle dernier. En 1975 précisément, le législateur français moderne a changé de regard quant aux causes conçues pour le divorce. Promulguant la loi $\mathrm{n}^{\circ}$ 75-617 du 11 juillet $1975^{3}$, il a tourné la page de la conception exclusive du divorce-sanction en intégrant d’autres cas de divorce pour rompre le mariage. Une loi dont l’objectif était la libérali-

\footnotetext{
${ }^{1}$ Parquet (M.), Droit de la famille, Bréal, 4e éd., 2014, p. 84.

${ }^{2}$ Il s'agit de la loi n 14-485 du 27 juillet 1884 (dite loi NAQUET), présentée par le parlementaire Alfred NAQUET, loi qui rétablit le divorce en France. Promulguée au J.O du 29 juillet 1884. http://www.justice.gouv.fr/art_pix/ Loi_retablissement_divorce_1884.pdf.

3 Entrée en vigueur le 1er janv. 1976.
} 
sation du droit du divorce 4 afin d'ouvrir plus grand les portes, d'admettre au divorce de nouvelles fournées de couples en difficultés ${ }^{5}$. La faute nest plus ainsi la seule cause de divorce mais un cas parmi d'autres ${ }^{6}$. Parmi ces cas, le législateur français a intégré la conception de divorce-remède ou divorce-faillite 7 pour cause objective ${ }^{8}$. La cause objective est définie, par le Doyen CARBONNIER, le directeur de cette loi, comme: celle qui paraît accorder le maximum à la liberté individuelle, puisqu'elle recouvre un état de rupture qui peut n'être qu'unilatéralement éprouvé. La législation française du divorce a été modifiée par la réforme de la loi n ${ }^{\circ}$ 2004-439 du 26 mai $2004^{10}$. Elle a maintenu le pluralisme des cas de divorce mais selon un nouveau regard. Nous nous intéressons ici à un type de divorce précis consacré par les deux lois citées, à savoir un divorce par la seule volonté unilatérale d'un époux sans s'appuyer sur une faute quelconque imputable au conjoint défendeur. Ce dernier subit un divorce imposé par la volonté de lautre époux non pour avoir commis une faute conjugale mais simplement car le demandeur du divorce ne souhaite plus continuer son mariage avec le conjoint défendeur. L’expérience française en l'espèce révèle deux modèles qui reflètent à quel point le législateur français a fait évoluer sa philosophie législative à légard de ce type de divorce. Dans un premier temps, le législateur de 1975 a intégré le divorce pour rupture de la vie commune (I). Trente ans après, le législateur de 2004 a présenté un autre modèle sous la forme du divorce pour altération définitive du lien conjugal (II).

\section{Le modèle du divorce pour rupture de la vie commune : dureté dans son mécanisme, sanction par les effets}

Le divorce pour rupture de la vie commune a constitué une innovation fondamentale de la réforme $e^{11}$ de 1975. Certes, l'idée nétait pas, à lépoque, de consacrer un droit au divorce selon toutes ses acceptions mais de traiter une réalité où la communauté de couple n'existe plus. Le législateur français a consacré ce type de divorce comme une solution pour celui qui vit depuis des années en concubinage afin de régulariser cette union et légitimer des enfants adultérins ${ }^{12}$.

Le divorce pour rupture de la vie commune est considéré comme un divorce pour cause objective mais d'une manière très restreinte. La cause objective, définie ci-dessus, a été rejetée, à

\footnotetext{
${ }^{4}$ Carbonnier (J.), «La question du divorce Mémoire à consulter», D, 1975, Chron XX, p. 117.

${ }^{5}$ Ibid.

${ }^{6}$ À côté du divorce pour faute jusque-là seul existant avant la loi de 1975, celle-ci a créé d'autres cas de divorce. Le divorce par consentement mutuel se caractérise par le divorce sur demande conjointe et le divorce sur demande acceptée. Plus encore, le divorce par la seule volonté sans besoin de se plaindre d'une faute quelconque a constitué une nouveauté à l'époque. Le divorce pour rupture de la vie commune regroupe quant à lui deux types : le divorce pour séparation de fait de plus de six ans et le divorce pour altération grave des facultés mentales.

${ }^{7}$ Les deux expressions employées par le rapporteur de cette loi, le Doyen J. CARbonnier, in «La question du divorce Mémoire à consulter», op. cit., p. 117 .

${ }^{8}$ Le professeur JACQUET voit que dans le divorce pour cause objective, ce qui est exigé n'est pas que le demandeur démontre au juge que le lien conjugal ne peut être maintenu car la vie commune est intolérable, mais c'est qu'il doit y être mis fin parce que le mariage n'est plus vécu comme tel. JACQUET (J.-M.), « Le rôle de la cause dans le nouveau droit français du divorce», RTD civ 1984, pp. 625 s.

9 Carbonnier (J.), « La question du divorce Mémoire à consulter», op. cit., p. 117.

${ }^{10}$ Publiée au Journal officiel du 27 mai 2004 et entrée en vigueur le 1er janvier 2005.

${ }^{11}$ Brazier (M.), Le nouveau droit du divorce, Éditions Apil, Versailles, p. 256. Adde Massip (J.), La réforme du divorce, (Commentaire des lois nos 75-617 et 75-618 du 11 juillet 1975 et des textes d'application), t. I, Répertoire du notariat Defrénois, p. 78.

${ }^{12}$ Carbonnier (J.), "La question du divorce Mémoire à consulter", op. cit., p. 119.
} 
l'époque, en tant que principe général'13. Il nétait pas envisageable, pour le législateur, d'adopter cette conception libérale donnée à la cause objective, afin de ne pas tomber dans le divorcerépudiation, tragédie pour l'épouse, sujet d'horreur pour [les] sociétés occidentales ${ }^{14}$. Le choix s'est alors porté sur un divorce-remède qui est concédé sous une forme atténuée ${ }^{15}$. Le divorceremède, traduit par le divorce pour rupture de la vie commune, a pris la forme de deux cas strictement précisés par la loi. Il s’agit du divorce pour séparation de fait depuis six ans (ancien art. 237 C. civ) et du divorce pour altération des facultés mentales (ancien art. 238 C. civ). Dans les deux cas, le divorce ne s'appuie pas sur une culpabilité de l'époux défendeur considéré comme innocent de toute faute conjugale. Celui qui demande ce divorce n'a pas à apporter la preuve d'une faute quelconque du défendeur ${ }^{16}$. Seulement la prise en considération d'un simple fait qui, depuis six ans, se prolonge $e^{17}$. Le divorce pour rupture de la vie commune est fondé sur des faits, non sur des fautes ${ }^{18}$, précise le Doyen Cornu. Le législateur de 1975 a conçu, pour les deux cas cités, un mécanisme par lequel la rupture de la vie commune peut être réalisée. Ce mécanisme a révélé des complications pour obtenir le divorce par des limites posées à la volonté unilatérale de la rupture (A). Plus encore, la loi a en quelque sorte sanctionnée le demandeur de ce divorce en mettant tous les effets du divorce à sa charge $(\mathrm{B})$.

\section{A. Divorce dur dans son mécanisme}

La loi du 11 juillet 1975 voyait le divorce pour rupture de la vie commune comme un remède $\grave{a}$ une situation autrement sans issue $e^{19} . \neq$ Elle a, cependant, adopté une conception stricte et restreinte pour rompre le mariage dans cette situation d'impasse. Pour prononcer le divorce en cas de rupture de la vie commune, il était indispensable que certaines conditions soient réunies (1). Mais cela nétait pas tout, le législateur a de plus imposé une clause de dureté pour mettre un frein au divorce et éviter l'automaticité de son prononcé (2).

\section{Conditions strictes à réunir}

Le législateur français de la loi de 1975 a retenu deux cas pour le divorce-remède, justement parce que ces cas peuvent être saisis à travers leur configuration matérielle, sans que le tribunal ait à faire d'investigations sur la profondeur de la désunion ${ }^{20}$. À cet effet, le législateur exigeait la réunion des conditions précises dans les deux cas admis par la loi.

Pour le premier, à savoir le divorce pour séparation de fait depuis six ans (ancien art. $237 \mathrm{C}$. civ), il fallait au minimum six ans de séparation de la communauté de vie entre les époux. Cette séparation repose sur deux éléments constitutifs: matériel et intellectuel ${ }^{21}$. Lélément matériel im-

\footnotetext{
${ }^{13}$ Ibid., p. 117 .

${ }^{14}$ Ibid., p. 115

${ }^{15}$ Ibid., p. 118.

${ }^{16}$ Furkel (F.), “La clause de dureté est-elle un mal nécessaire?”, D. 1977, Chro X, p. 83.

${ }^{17}$ Monsallier (B.), “Le divorce pour rupture de la vie commune (Loi du 11 juillet 1975)", RTD. civ, 1980, p. 267.

${ }^{18}$ Cornu (G.), La réforme du divorce (étude de la loi du 11 juillet 1975), Les cours de droit, Paris, 1976, p. 163.

${ }^{19}$ Rubellin-Devichi (J.), "La diminution de l'importance du mariage "L'admission du divorce par volonté unilatérale” ", in Mariage et famille en question, Tome 1, Dir. R. Nerson, Éditions du C.N.R.S, 1978, p. 89.

${ }^{20}$ Carbonnier (J.), "La question du divorce Mémoire à consulter", op. cit., p. 117.

${ }^{21}$ La Cour de cassation a affirmé ces deux éléments, en réaffirmant ce que la cour d’appel de Paris a rendu : "Mais attendu que l>arrêt, après avoir rappelé que l>art. 237 du Code civil n`effectue aucune distinction quant aux circonstances ayant accompagné la séparation des époux, énonce exactement quil suffit, pour que les conditions prévues par la loi soient remplies, que la communauté de vie, tant matérielle qu>affective, ait cessé entre les conjoints [...] “. 
plique nécessairement une résidence séparée pour révéler le fait d’absence de cohabitation. Cet élément constitue le critère objectif qui servira de base de départ à l'appréciation du magistrat pour l'examen de la recevabilité de la requête ${ }^{22}$. Néanmoins, l'absence de cohabitation comme élément matériel ne suffit pas à lui tout seul pour réaliser la séparation de vie commune exigée par le texte. Il faut ajouter un autre élément considéré comme l'âme de la séparation ${ }^{23}$, à savoir léelément intentionnel. La rupture entre les époux doit être voulue par au moins l’un d'eux. Quant à la cessation de la communauté de vie d’au moins six ans, ce délai long et nécessaire est considéré comme présomption de la faillite irrémédiable du couple $e^{24}$. Il se caractérise par deux points : la continuité et la date. La séparation ne doit pas avoir été interrompue par une réconciliation réelle $^{25}$ et le délai de six ans court à partir de la présentation de la requête26. Par ces exigences, il est clair que le législateur français a voulu constater une situation claire dans le couple par la cessation de la communauté de vie pendant une durée prolongée. Autrement dit, il noffre pas le divorce pour séparation de fait pour les époux qui vivent ensemble dans la mésentente ${ }^{27}$.

Pour le second cas, à savoir le divorce pour altération des facultés mentales (ancien art. 238 C. civ), cette altération doit être grave et avoir duré six ans. Aucune communauté de vie ne doit plus subsister entre les époux. La loi n’a pas exigé une séparation de fait pour ce cas. Elle exige la cessation de communauté de vie et non la séparation des époux ${ }^{28}$. Mais, même si l'incurabilité de la maladie nest pas une condition, dans ce cas de divorce, la loi exigeait, tout de même, que selon les prévisions les plus raisonnables, aucune communauté de vie ne puisse se reconstituer dans l'avenir ${ }^{29}$. Enfin, un lien de causalité entre l'altération des facultés mentales et la cessation de communauté de vie doit exister ${ }^{30}$.

Les conditions exigées par le législateur étaient soumises au pouvoir discrétionnaire du juge. Celui-ci avait un rôle considérable pour apprécier la durée de six ans et la gravité de la maladie, constatées par un rapport médical (ancien art. 1125 C.P.C.).

Mais les exigences visées par la loi ne suffisaient pas pour garantir le prononcé du divorce souhaité par une seule volonté. Même si les conditions étaient réunies, le divorce ne pouvait être automatiquement prononcé en raison de la clause de dureté.

\section{Intégration de la clause de dureté}

Le législateur ne se contentait pas dexiger des conditions restreintes et complexes pour permettre le divorce pour rupture de la vie commune, il a encore ajouté un autre obstacle devant la volonté unilatérale de rupture. Il s’agissait de la clause de dureté qui pouvait déjouer le pronon-

Cass. 2e civ., 30 janvier 1980, JCP G, 1981. II, J, 19521, Obs. Lindon (R.); Rép. Defrénois 1980, nº 65, art. 32421, pp. 1206s, note MASsIP (J.).

${ }^{22}$ Monsallier (B.), "Le divorce pour rupture de la vie commune (Loi du 11 juillet 1975)", op. cit., p. 272.

${ }^{23}$ Cornu (G.), La réforme du divorce (étude de la loi du 11 juillet 1975), Les cours de droit, Paris, 1976, p. 176.

${ }^{24}$ Monsallier (B.), "Le divorce pour rupture de la vie commune (Loi du 11 juillet 1975)", op. cit., p. 274.

${ }^{25}$ Lindon (R.), La nouvelle législation sur le divorce et le recouvrement public des pensions alimentaires (lois du 11 juillet 1975 : Analyse et commentaire), Litec, 1975, p. 13.

${ }^{26}$ Massip (J.), La réforme du divorce, (Commentaire des lois nos 75-617 et 75-618 du 11 juillet 1975 et des textes d'application), t. I, op. cit., p. 73 .

${ }^{27}$ Connu (G.), La réforme du divorce (étude de la loi du 11 juillet 1975), op. cit., p. 177.

${ }^{28}$ Massip (J.), La réforme du divorce, (Commentaire des lois nos 75-617 et 75-618 du 11 juillet 1975 et des textes d'application), t. I, op. cit., p. 78.

${ }^{29}$ Ancien art. 238 du C. civ.

${ }^{30}$ Massip (J.), La réforme du divorce, (Commentaire des lois nos 75-617 et 75-618 du 11 juillet 1975 et des textes d'application), t. I, op. cit., p. 79. 
cé du divorce. Par cette clause le juge pouvait rejeter ce prononcé, si le défendeur abandonné établissait que le divorce aurait, soit pour lui, compte tenu notamment de son âge et de la durée du mariage, soit pour les enfants, des conséquences matérielles ou morales diune exceptionnelle dureté $e^{31}$. Plus encore, le juge a le droit de rejeter d'office la demande de rupture si le divorce risque d'avoir des conséquences trop graves sur la maladie du conjoint ${ }^{32}$. Elle laisse ainsi au juge un pouvoir d'appréciation et évite toute automaticité dans le prononcé du divorce ${ }^{33}$. En intégrant cette clause, la loi a institué une véritable "défense au fond", un moyen qui [...] tend à faire rejeter, au fond, la prétention de l'adversaire ${ }^{34}$. Le législateur français a voulu, en effet, établir une différence indiscutable entre la répudiation et le divorce pour rupture de la vie commune auquel on a reproché une similitude avec la répudiation. Les aspects discrétionnaire et automatique caractérisant la répudiation sont absents du divorce pour rupture de la vie commune. Avec la clause de dureté, et compte tenu des circonstances, le juge avait la possibilité d`intervenir et d`imposer le maintien du lien conjugal ${ }^{35}$. L'admission ou le rejet de la clause de dureté dépend d'une appréciation plus morale que juridique, où les juges du fond exercent en principe un pouvoir souverain ${ }^{36}$. Rien ne garantit, alors, le prononcé du divorce avec la clause de dureté qui peut légitimement déjouer la volonté unilatérale du demandeur de la rupture. L'intégration de la clause d'une exceptionnelle dureté révèle bien que le prononcé du divorce pour rupture de la vie commune n'était pas de droit ${ }^{37}$. Cette conclusion se renforce par les lourds effets que ce divorce engendre pour le demandeur.

\section{B. Divorce sanctionné par les effets}

La voie très restreinte que le législateur a ouverte à la volonté unilatérale de rupture était au prix d'effets très lourds pour le demandeur du divorce. Comme une partie de la doctrine lıa décrit, cétait un divorce assumé par le demandeur ${ }^{38}$. Celui-ci devait en effet assumer toutes les charges face à son conjoint lésé. Plus encore, le demandeur se trouvait privé de tout avantage. Les énumérations des lourdes conséquences du divorce (1) révèlent bien comment le divorce pour rupture de la vie commune a été considéré comme une sanction pour celui qui en prenait l'initiative (2).

${ }^{31}$ Alinéa 1 de l'ancien article 240 du C. civ.

${ }^{32}$ Alinéa 2 de l'ancien article 238 du C. civ.

${ }^{33}$ Massip (J.), La réforme du divorce, (Commentaire des lois nos 75-617 et 75-618 du 11 juillet 1975 et des textes d'application), t. I, op. cit., p. 80.

34 Cornu (G.), La réforme du divorce (étude de la loi du 11 juillet 1975), op. cit., p. 206.

35 Furkel (F.), “La clause de dureté est-elle un mal nécessaire ?”, op. cit., p. 84. Adde Brazier (M.), Le nouveau droit du divorce, Éditions Apil, Versailles, p. 256.

${ }^{36}$ Carbonnier (J.), Droit civil “Introduction, les personnes, la famille, l'enfant, le couple”, PUF, Paris, 2e éd., 2017, Vol. I., p. 1307.

37 Garrigue (J.), Les devoirs conjugaux "Réflexion sur la consistance du lien matrimonial”, Éditions PanthéonAssas II, Collection Thèses, 2012, p. 366.

${ }^{38}$ Massip (J.), La réforme du divorce, (Commentaire des lois nos 75-617 et 75-618 du 11 juillet 1975 et des textes d'application), t. I, op. cit., p. 69. 


\section{Régime spécifique sévère pour les effets du divorce}

Compte tenu de la dureté de son choix en adoptant le divorce pour rupture de la vie commune, le législateur français s'est appuyé sur de lourds effets pécuniaires pour atténuer la dureté de ce type de divorce. Le demandeur devait en effet :

- Perdre certains avantages: absence de tout droit à prestation compensatoire, déchéances des donations et avantages matrimoniaux (anc. art. 269 C. civ).

- Supporter les charges du divorce (anc. art. 239 C. civ) ${ }^{39}$.

- Assumer le devoir de secours subsistant au profit du conjoint abandonné (anc. art. 270 et 281 C. civ). En cas de divorce pour altération des facultés mentales, le devoir de secours couvre tout ce qui est nécessaire au traitement médical du conjoint malade (anc. art. 281-1 C. civ).

La loi a en outre donné à l'époux lésé qui subit le divorce, considéré comme innocent, le droit de conserver les donations et avantages matrimoniaux (anc. art. 269-2 C. civ) et la protection de son logement (anc. art. 285-1 C. civ). Et sur le plan personnel, si ce conjoint était la femme, elle avait le droit de continuer à porter le nom de son mari (anc. art. 264-2 C. civ) ${ }^{40}$.

\section{Divorce au prix d'une sanction automatique}

Lénumération des effets financiers reflète le regard du législateur français envers le divorce pour rupture de la vie commune à l'époque. L`époux qui a pris linitiative du divorce pour rupture de la vie commune est considéré comme responsable de la dissolution du mariage ${ }^{41}$. Dans ce divorce, l'époux demandeur "assume la responsabilité de son divorce" ${ }^{42}$. Cette responsabilité traduite par ces lourdes conséquences révèle bien que le législateur de 1975 ne s'est pas tout à fait détaché de la conception de faute même dans le divorce pour rupture de la vie commune ${ }^{43}$. Le demandeur de ce divorce se trouvait sanctionné automatiquement par les effets du divorce, ce qui le mettait dans un cas similaire à un époux fautif. Le divorce pour rupture de la vie commune traduisait une issue légale qui place l'époux demandeur dans une situation analogue à celle du conjoint aux torts exclusifs de qui le divorce a été prononcé 44 . La loi de 1975 a créé une cause de divorce qui met automatiquement les torts à la charge du demandeur ${ }^{45}$. Lancien article 265 du C. civ prévoyait clairement: Le divorce est [...] aussi réputé prononcé contre l'époux qui a pris

\footnotetext{
39 Le second alinéa de l'ancien art. 239 C. civ. prévoyait également: “Dans sa demande, [l'époux qui demande le divorce pour rupture de la vie commune] doit préciser les moyens par lesquels il exécutera ses obligations à l'égard de son conjoint et des enfants".

${ }^{40}$ Pour ces informations et plus, voir : Brazier (M.), Le nouveau droit du divorce, op. cit., pp. 269s; BLESsy (J.-C.), Le principe du divorce objectif étudié dans ses rapports avec la faute en droits français et allemand comparés, th. Nice, 1989, pp. 199s.

${ }^{41}$ Massip (J.), La réforme du divorce, (Commentaire des lois nos 75-617 et 75-618 du 11 juillet 1975 et des textes d'application), t. I, op. cit., p. 210.

${ }^{42}$ Cornu (G.), La réforme du divorce (étude de la loi du 11 juillet 1975), op. cit., p. 170.

${ }^{43}$ V. sur ce sujet : Furkel (F.), "La faute dans le divorce en droit français et allemand", RIDC, Vol. 34, n 4, octobredécembre 1982, pp. 1153s ; Jung (B.), "Le divorce remède ou la place faite dans le nouveau droit du divorce en France et en République fédérale d'Allemagne”, JCP G, 1979, I, 2940.

${ }^{44}$ Connu (G.), La réforme du divorce (étude de la loi du 11 juillet 1975), op. cit., p. 170.

${ }^{45}$ Lindon (R.), La nouvelle législation sur le divorce et le recouvrement public des pensions alimentaires: (lois du 11 juillet 1975 : Analyse et commentaire), op. cit., p. 12.
} 
l'initiative du divorce lorsqu'il a été obtenu en raison de la rupture de la vie commune ${ }^{46}$. En effet, le législateur a déplacé l'auteur de la faute, selon les différents cas de divorce. Dans le cas du divorce pour faute, le défendeur est lauteur admis de la faute, mais dans le divorce pour rupture de la vie commune, le demandeur de ce divorce est l'auteur de la faute ${ }^{47}$. La situation du demandeur du divorce pour rupture de la vie commune peut être expliquée par le fait que ce demandeur se trouve devant un divorce contre soi-même (Tout se passe comme si l'époux demandait et obtenait le divorce contre lui-même ${ }^{48}$. Si la cause du divorce ne s'appuie pas sur la faute du défendeur, ses conséquences assumées par le demandeur mettent ce dernier en posture de fautif. La volonté du conjoint peut être à l'origine d'une demande en divorce mais si elle lıobtient, elle sera sanctionnée par de lourdes conséquences. Ainsi la notion de responsabilité est réintroduite dans les conséquences du divorce objectiff9. Le divorce pour rupture de communauté de vie est prononcé contre un époux considéré comme innocent qui ne le souhaite pas et imposé par la volonté unilatérale d’un époux présumé fautif. Certes, il est logique que le demandeur du divorce par la seule volonté paie le prix de sa liberté. Néanmoins, le prix que le demandeur du divorce paie pour rupture de la vie commune n'est pas un simple prix, mais une sanction lourde, semblable à celle qui serait appliquée en cas de faute grave. La conception stricte de ce divorce pour rupture de la vie commune n’a pas rencontré, au fil du temps, le moindre succès. Ce type de divorce était quasiment inexistant dans la pratique française ${ }^{50}$ du fait qu'il engendrait des conséquences pécuniaires très lourdes ${ }^{51}$. Son utilité, du fait d'un taux n'ayant jamais excédé les $1,5 \%{ }^{52}$, a fait lobjet de très nombreuses critiques ${ }^{53}$. La loi de 1975 a sans doute marqué une première étape dans la législation française moderne, visant à élargir la conception du divorce. Une seconde étape a débuté par la réforme de 2004, avec pour but premier de réformer le divorce pour rupture de la vie commune. Ce dernier a été transformé en divorce pour altération définitive du lien conjugal.

${ }^{46}$ Le premier alinéa de l'ancien article 265 du C. civ prévoyait : "Le divorce est réputé prononcé contre un époux sil a eu lieu à ses torts exclusifs".

47 Alati (A.), "La rupture du mariage par la volonté unilatérale de liépouse : Étude de législation française et de législation islamique appliquée en pays arabes", Volume I, Éditions universitaires européennes, 2018, p.347.

${ }^{48}$ Cornu (G.), La réforme du divorce (étude de la loi du 11 juillet 1975), op. cit., p. 170.

49 Jung (B.), "Le divorce remède ou la place faite dans le nouveau droit du divorce en France et en République fédérale d'Allemagne", op. cit., $\mathrm{n}^{\circ} 13$.

${ }^{50}$ Dekeuner-Défossez (F.), Rénover le droit de la famille (Propositions pour un droit adopté aux réalités et aux aspirations de notre temps), Rapport au Garde des Sceaux, ministre de la Justice, La Documentation Française, 1999, p. 114.

${ }^{51}$ Rubellin-Devichi (J.), "Les grandes réformes en cours dans le droit de la famille en France”, in Droit comparé des personnes et de la famille, Bruylant, Bruxelles, 1998, pp. 680s.

${ }^{52}$ Le ministère de la Justice a réalisé en 1996 une enquête exceptionnelle fondée sur l'analyse d'un large échantillon de jugements de divorces rendus en 1994. Elle montre que le divorce pour rupture de la vie commune ne présente que $1,5 \%$ du total des divorces. Ce chiffre n'est pas différent des autres années. Le divorce pour rupture de la vie commune étaient en 1994 de 1726 (sur un total de 116 072), en 1995 de 1681 (total 120 027), en 1996 de 1783 (total 117 716), en 1997 de 1877 (total 116 617), en 1998 de 1942 (total 116 831), en 1999 de 1953 (total 117 494), en 2000 de 1812 (total 114 620), en 2001 de 1512 (total 113 618), en 2002 de 1616 (total 117 005), en 2003 de 1505 (total 126388 ). Annuaire statistique de la justice, La Documentation française, Paris, édition : 2000, pp. 79s, 2001, pp. 81s, 2005, p. 89 .

${ }^{53}$ V. sur ce point : DeKeuwer-Défossez (F.), Rénover le droit de la famille (Propositions pour un droit adopté aux réalités et aux aspirations de notre temps), op. cit., p. 114. 


\section{Le modèle du divorce pour altération définitive du lien conjugal : souplesse dans son mécanisme, absence de sanction par les effets}

Léchec, pour ainsi dire, du divorce pour rupture de la vie commune, d'une part, et les inconvénients du divorce pour faute, d’autre part, ont incité le législateur français à réfléchir à réformer le divorce pour rupture de la vie commune. La stratégie du législateur de la réforme de 2004 visait à trouver un compromis entre le maintien du divorce pour faute et l’ouverture à la volonté unilatérale d`un divorce sans faute imputable au conjoint ${ }^{54}$. Son choix sest porté sur l'instauration d'un divorce par une seule volonté, totalement hors de la conception de faute et qui peut, de plus, être présenté comme une alternative au divorce pour faute 55 . Pour atteindre cet objectif, la réforme de 2004 a adopté une forme de divorce plus souple dans son mécanisme (A) et sans pénalisation par les effets financiers (B).

\section{A. Divorce plus souple dans son mécanisme}

La principale modification que la réforme de la loi de 2004 a apportée à la rupture unilatérale se concentre sur la modalité de ce genre de divorce. Le législateur a radicalement bouleversé la conception de divorce pour la rupture de la vie commune. Plus de souplesse dans les dispositions qui gouvernent ce divorce était indispensable pour qu>il soit plus attractif et dynamique ${ }^{56}$. Lactuel article $238 \mathrm{du}$ C. civ $^{57}$ a donné un nouveau visage à la rupture du mariage pour cause objective. Le législateur a unifié le divorce pour altération en supprimant l`altération des facultés mentales. Que le conjoint soit malade ou non, le divorce pour altération soumet tout le monde aux mêmes règles. La réforme de 2004 a prévu deux hypothèses pour la demande présentée dans le cadre du divorce pour altération définitive du lien conjugal, à savoir soit une demande à titre principal soit à titre reconventionnel. Le premier type de demande concerne une altération conditionnée par une séparation mais facilitée par rapport à lrancienne loi de 1975 (1). Le second type traite une altération même sans séparation (2). Dans les deux hypothèses, la finalité du législateur était d’assouplir les dispositions pour obtenir le divorce plus facilement que sous lancienne loi.

\section{Altération conditionnée par une séparation facilitée}

Pour faciliter la demande du divorce pour altération définitive à titre principal, le législateur s’est appuyé sur deux points : le premier concerne la souplesse des conditions exigées vis-à-vis de

\footnotetext{
${ }^{54}$ Comme le conseiller J. Massip l'indique : "Le législateur espère que cette nouvelle conception du divorce, [à savoir le divorce pour altération définitive du lien conjugal] sera de nature à détourner un grand nombre de conjoints du divorce pour faute, qui, dans l'esprit de la loi nouvelle, devrait être réservé aux manquements les plus graves aux obligations du mariage", in Le nouveau droit du divorce, Defrénois, 2005, p. 32.

${ }^{55}$ Himmi-Menr abi (M.), La dédramatisation du divorce dans la législation contemporaine, op. cit., p. 182.

${ }^{56}$ Aujourd'hui, le divorce pour altération définitive du lien conjugal rencontre un succès. Il a pris sa place parmi les autres formes de divorce avec $13 \%$ des divorces prononcés en 2013 et a même devancé le divorce pour faute qui ne représentait plus que 8\% des divorces la même année. V. Ministère de la Justice, Divorces prononcés en 2013, Ministère de la Justice/SG/SDSE. http://www.justice.gouv.fr/art_pix/stat_article_ruptures_union_Internet.pdf.

${ }^{57}$ L’article 238 prévoit: "L`altération définitive du lien conjugal résulte de la cessation de la communauté de vie entre les époux, lorsquils vivent séparés depuis deux ans lors de liassignation en divorce. Nonobstant ces dispositions, le divorce est prononcé pour altération définitive du lien conjugal dans le cas prévu au second alinéa de liarticle 246, dès lors que la demande présentée sur ce fondement est formée à titre reconventionnel".
} 
l'ancien divorce pour rupture de la vie commune (a). Le second point se porte sur la suppression de la clause de dureté (b).

\section{a. Conditions assouplies}

La demande de divorce pour altération définitive du lien conjugale doit répondre à deux exigences prévues dans le texte législatif: cessation de la communauté de vie, et ce pendant au moins deux ans.

Cessation de la communauté de vie: sur ce point les deux éléments exigés sous l`empire de l ancien divorce pour rupture de la vie commune, à savoir un élément matériel et un élément intentionnel ${ }^{58}$, restent les mêmes. L'intention de se séparer est donc nécessaire, de la part d'un époux au moins. En somme, la séparation doit être voulue ${ }^{59}$. La nécessité de ces deux éléments signifie l'inefficacité de la séparation affective comme motif légal du divorce pour altération définitive du lien conjugal ou pour demander ce type de divorce. Une simple séparation affective ne suffit pas à remplir le cas voulu par l'alinéa 1 de l'article 238 du C. civ, car la vie séparée érigée en cause de divorce suppose la non-cohabitation ${ }^{60}$. La séparation affective et intellectuelle sans séparation matérielle ne peut apporter la preuve de la cessation de la communauté de vie $e^{61}$, pour convaincre le juge.

Deux ans de séparation: Selon les dispositions de l'alinéa 1 de l'article 238, les deux époux doivent vivre séparés depuis deux ans lors de l'assignation en divorce. Par cette durée, la loi de 2004 a pris parti pour la nécessité d’exiger un délai de séparation62. La nouveauté, par rapport à l'ancienne loi, réside ici dans deux points : le premier est la réduction du délai de six à deux ans. Cette durée préfixée de deux ans ${ }^{63}$ doit être continue ${ }^{64}$ (ni interrompue, ni raccourcie) ${ }^{65}$ entre le moment où la vie commune des époux a cessé et l'assignation en divorce ${ }^{66}$. Le second

\footnotetext{
${ }^{58}$ Pour la loi actuelle, la circulaire du 23 novembre 2004 a traité la condition de la cessation de la communauté de vie en précisant que : "Cette condition revêt, à l'instar de ce que la jurisprudence exigeait en matière de divorce pour rupture de la vie commune, un aspect à la fois matériel (l'absence de cohabitation) et psychologique (la volonté de rupture)". Circ. min. ${ }^{\circ} \mathrm{CIV} / 16 / 04,23$ nov. 2004

59 Courbe (P.), Le divorce, Dalloz, Connaissance du droit, 4e éd., 2004, p. 25.

${ }^{60}$ Cornu (G.), Droit civil la famille, Domat droit privé, Montchrestien, Paris, 9e éd., 2006, p. 545.

${ }^{61}$ Même si "la cohabitation matérielle des époux ne signifie pas nécessairement quil existe ou subsiste entre les époux une communauté de vie : la cohabitation matérielle peut être exclusive de toute survie de la communauté affective et intellectuelle et ne continuer que pour des raisons de commodité ou même de nécessité matérielle". Larribau-Terneyre (V.), "Sur la notion de la communauté de vie des époux", note sous : CA Versailles, 2e ch., 31 mars 2011, n 10-02387. Dr. Fam, 2011, n 6, Comm. 94, pp. 30 s.

${ }^{62}$ Dans un projet de loi présenté en 2001 par le député Colcombet, il n'était pas demandé de séparation préalable à la demande de divorce pour cause de rupture irrémédiable du lien conjugal. Par conséquent, il n'était pas prévu de délai pour envisager le divorce. Dans ce cas, l'époux demandeur invoquait le caractère irrémédiable de la rupture du lien conjugal, mais seulement à l'issue d'une période de réflexion fixée par le juge. $h t t p: / / w w w$. assemblee-nationale.fr/11/propositions/pion3189.asp

${ }^{63}$ Rapport pour la Commission des lois, Sénat $n^{\circ}$ 120, présenté par le Sénateur P. GÉLARD, sur le projet de loi relatif au divorce, annexe au procès-verbal de la session du 17 décembre 2003. http://www.senat.fr/rap/lo3-120/ lo3-1201.pdf

${ }^{64}$ Courbe (P.), Le divorce, op. cit., p. 24 ; Massip (J.), Le nouveau droit du divorce, Defrénois, 2005, p. 28.

${ }^{65}$ P. GÉLARD, Rapport pour la Commission des lois, op. cit., Rapport Sénat, $\mathrm{n}^{\circ}$ 120, op. cit.

${ }^{66}$ Sans doute, la détermination du point de départ ne pose aucune difficulté quant à la séparation juridique. La date de la décision qui se prononce sur la résidence séparée, le jugement de séparation de corps ou l'ordonnance de non conciliation, fixera aisément ce point ; "lıépoux demandeur est en mesure de s`appuyer sur une séparation juridique, laquelle se prouve sans difficulté par la production de la décision judiciaire qui a statué sur la résidence 
point de nouveauté, qui constitue un changement radical par rapport à l'ancienne loi de 1975, réside dans l'appréciation de ce délai. Celui-ci ne court plus à partir du jour de la présentation de la requête en divorce mais à partir de la date de lıassignation en divorce comme auparavant ${ }^{67}$. Il s'agit de la seconde phase de la procédure ${ }^{68}$. À cet égard, la séparation intervenue avant ou après la requête initiale en divorce, et celle intervenue après l'ordonnance de non conciliation, dès lors que cette séparation présente un caractère continu pendant les deux années précédant l assignation ${ }^{69}$, peuvent ainsi indifféremment être prises en compte. Ce mode d'appréciation du délai révèle que le législateur a voulu ouvrir cette forme de divorce non seulement à des conjoints déjà séparés au moment de la requête initiale mais aussi à des époux non encore séparés $^{70}$. Il traite le cas du couple déjà séparé tout en octroyant le droit à un conjoint de préparer sa séparation en toute sécurité. Dans ce cas, un époux non encore séparé, ou qui vient juste de se séparer, peut valablement déposer une requête en divorce (art. 251 C. civ.), sans mentionner dès à présent le cas de divorce qui sera utilisé ${ }^{71}$. Cette requête initiale permet d’obtenir des mesures provisoires et dans le cadre de l'ordonnance de non-conciliation ${ }^{72}$, le magistrat conciliateur accorde l'autorisation de résider séparément. Il ne reste plus ensuite à l'époux demandeur qu>à patienter deux années pour remplir la condition de délai exigée et déposer une assignation fondée, si tel est toujours son choix, sur l>altération définitive du lien conjugal73. Alors, l'époux qui souhaite divorcer peut déposer sa requête initiale sans attendre dêtre séparé depuis deux ans ${ }^{74}$ dans la mesure où le délai peut être entièrement acquis entre l'ordonnance de non-conciliation et l'assignation ${ }^{75}$. La voilà la répudiation à la française ${ }^{76}$ qui n'a pas besoin, dans ce cas, d’une cause déterminée pour être prononcée. Pour des considérations personnelles, un époux peut donc engager ce type de divorce, il lui suffit de remplir les conditions ou plutôt la condition de délai. Le juge n`a pas le droit de s`opposer à ce divorce même en cas de défaut d'expiration du délai de deux ans sauf si l>époux défendeur ne comparaît pas ${ }^{77}$. Dès lors que la preuve de la séparation de deux ans, au moment de l'assignation, est rapportée, il peut seulement prononcer le divorce $e^{78}$. Ce cas de divorce, voulu par le législateur, a incité ce dernier à modifier le délai des mesures provisoires. Celles-ci sont devenues, depuis la loi de 2004, valables pendant trente mois

\footnotetext{
séparée". Cependant, la détermination du début de la séparation de fait peut s'avérer plus délicate dans le cas où les deux époux sont en désaccord quant à la date exacte de leur séparation. Mais, "plus la durée de la séparation est longue, plus la preuve est aisée à apporter". V. ces informations successivement dans : DAVID (S.), "Divorce altération : le point de départ du délai de séparation se prouve par tous moyens”, Obs. sous Cass. 1re civ., 25 novembre 2009, $\mathrm{n}^{\circ}$ 08-17.117, AJ Fam, 2010, p. 136 ; "Seules diéventuelles discussions sur la survenance d`une réconciliation postérieure peuvent alors surgir”, précisent certains spécialistes. Claux (P.-J.), David (S.) et autres, Droit et pratique du divorce, Dalloz Référence, Dalloz, 2e éd., 2013, p. 35.

67 Il s'agit de la loi de 1975.

${ }^{68}$ Bonnet (V.), Droit de la famille, manuel, Larcier, 5e éd., 2015, p. 252.

${ }^{69}$ C'est l'explication des auteurs de la circulaire du 23 novembre 2004. Circ. min. n CIV/16/o4, 23 nov. 2004.

${ }^{70}$ Claux (P.-J.), David (S.) et autres, Droit et pratique du divorce, op. cit., pp. 33 s.

${ }^{71}$ Murat (P.) (dir.), Droit de la famille, Droit de la famille, Dalloz-action, 7e éd., 2016-2017, p. 314.

$7^{72}$ Roy (O.), Droit de la famille, Collection Montesquieu, Archétype 82, Paris, 2014, pp. 87s.

73 Claux (P.-J.), David (S.) et autres, Droit et pratique du divorce, op. cit., pp. 33 s.

74 "L’époux demandeur peut entamer la première phase de la procédure (requête initiale) alors même que le délai n>est pas entièrement écoulé “. Bonnet (V.), Droit de la famille, op. cit., p. 252.

75 Droit de la famille 2016-2017, mémento pratique, Éditions Francis LEFEBVRE, p. 180.

${ }^{76}$ Alati (A.), "La rupture du mariage par la volonté unilatérale de liépouse: Étude de législation française et de législation islamique appliquée en pays arabes", Volume I, op. cit., p. 422.

77 L'article 1126 du CPC (modifié par Décret n² 2008-484 du 22 mai 2008) prévoit que "le juge ne peut relever d'office le moyen tiré $d u$ défaut d'expiration du délai de deux ans prévu au premier alinéa de l'article 238 du Code civil" sauf si l'époux défendeur ne comparaît pas. Ce moyen de défense appartient, en effet, à l'époux défendeur.

${ }^{78}$ Gouttenoire (A.), "Les nouvelles procédures de divorce”, Rép. Defénois, 2005, n 7, Doct., art. 38137, p. 558.
} 
selon l'article $1113 \mathrm{CPC}^{79}$. Tout cela pour permettre au conjoint qui vit encore avec son époux d’engager la procédure, pour qu'il puisse légitimement créer sa séparation de deux ans. Par cette stratégie, à savoir le délai décompté à la date de l'assignation, le législateur français a orienté sa philosophie vers le divorce unilatéral en écrivant une nouvelle page dans l'histoire du divorce en France. Une page qui efface la stigmatisation d'un divorce par la volonté unilatérale sans cause déterminée et qui permet même de le préparer pas à pas. La finalité de la réforme de 2004 de donner plus de liberté à la volonté unilatérale d`un époux pour rompre le mariage a été renforcée, en outre, par la suppression de la clause de dureté.

\section{b. Suppression de la clause de dureté}

Parmi les dispositions ciblées par la réforme de 2004, pour faciliter lıaccès au divorce pour altération définitive du lien conjugal, figure la suppression de la clause de dureté. Celle-ci, considérée comme une sorte de véto à la rupture ${ }^{80}$, a été retirée pour s'orienter vers l>automatisation du prononcé du divorce. Par cette suppression, le dernier obstacle auquel pouvait se heurter le désir de rompre a ainsi disparu ${ }^{81}$. Le conjoint contre lequel le divorce est demandé ne peut plus réellement s'opposer à la volonté du demandeur du divorce. Celui-ci imposera sa volonté de rupture à un conjoint innocent qui ne veut pas la rupture et quelles que soient les conséquences de ce divorce. La clause d'exceptionnelle dureté, constituant un moyen de défense de fond, pouvait, malgré sa rareté ${ }^{82}$, faire obstacle au désir du demandeur ou permettait parfois de faire échec au divorce ${ }^{83}$. Tout au moins, elle donnait une marge d’opposition qui atténuait la liberté donnée à la volonté unilatérale du demandeur. Le juge n’a plus de pouvoir discrétionnaire pour juger l'opportunité de prononcer le divorce. Une fois réunies les conditionnes exigées par la loi, il ne peut plus que prononcer le divorce. La suppression de cette clause révèle une preuve éclatante du recul de l'influence de la religion, spécialement catholique, dans l'édiction des normes applicables aux relations familiales ${ }^{84}$. Pour le législateur, cette suppression était nécessaire car le mariage peut être préservé lorsqu'il a un sens. S'il y a des conséquences graves pour l'un des conjoints, la réponse [n'est pas] le maintien de force du mariage ${ }^{85}$. La société ne peut plus concevoir qu'un époux, qui veux mettre fin au mariage sans avoir ni l`accord de l`autre, ni un grief à son encontre, doive rester marié contre son gré86. Cela révèle, sans doute, l'évolution de la philosophie législative de la réforme de 2004 à l’égard du concept même de mariage.

\footnotetext{
79 Cet article prévoit que "Dans les trois mois du prononcé de l>ordonnance, seul lıépoux qui a présenté la requête initiale peut assigner en divorce. En cas de réconciliation des époux ou si linstance n>a pas été introduite dans les trente mois du prononcé de l>ordonnance, toutes ses dispositions sont caduques, y compris l>autorisation dintroduire linstance.

${ }^{80}$ Koumdadji (A.), La sécularisation de la répudiation: ou l'avènement de la rupture unilatérale et discrétionnaire en droit français, Les éditions du Cerf P, 2015, p. 201.

${ }^{81}$ Garrigue (J.), Les devoirs conjugaux "Réflexion sur la consistance du lien matrimonial”, op. cit., p. 366.

${ }^{82}$ Le professeur GARÉ, en commentant l'arrêt rendu par la deuxième chambre civile de la Cour de cassation le 13 mars 2003, a remarqué "qu>en près de trente ans, la clause de dureté tirée des convictions religieuses n>a pas été retenue plus dıune dizaine de fois". GARÉ (Th.), "Pour un réexamen des règles applicables à la clause de dureté fondée sur les règles religieuses", Analyse pour Cass. 2e civ., 13 mars 2003, $\mathrm{n}^{\circ}$ 01-14.616, RJPF, 2003, $\mathrm{n}^{\circ}$ 6, p. 19; S. D, "La clause d'exceptionnelle dureté demeure exceptionnellement admise", note sous CA Paris, 24e ch. A, 11 juin 2003, RG n²002/02053, AJ Fam, 2003, pp. 384s.

${ }^{83}$ Larribau-Terneyre (V.), La réforme du divorce: premier bilan à mi-parcours, Dr. Fam, 2004, n 3 , Chron. 6, p. 5 .

${ }^{84}$ Fornerod (A.), "Religion et famille en droit interne", in Droit français des religions, Dir. Messner (F.), PrÉLOT (P.-H.) et Woehrling (J.-M.), avec la contribution d'Isabelle Riassetto, Traités, Lexis Nexis, Paris, 2013 , p. 1016.

85 Granet-Lambrechts (F.), "Les nouveaux cas de divorce”, AJ Fam., 2004, p. 207.

${ }^{86}$ Delecraz (Y.), “Le projet de réforme du divorce”, Rép. Defrénois, 15 mai 2004, n 9, art. 37935, p. 647. 


\section{Altération sans même séparation du couple}

Les dispositions de l'alinéa 2 de l'article $238^{87}$ recouvrent une situation très différente de celle visée au premier alinéa du même article ${ }^{88}$. Le deuxième alinéa de l`article cité dispense de la preuve de la cessation de la communauté de vie de deux ans, si la demande fait suite à une demande de l>autre époux pour faute ${ }^{89}$. Cet alinéa recouvre le cas où une demande en divorce pour faute est présentée principalement et que le défendeur forme une demande reconventionnelle en divorce pour altération définitive du lien conjugal. Dans ce cas, si le juge rejette la demande de divorce pour faute, il prononce le divorce pour altération sans que le respect des conditions de l'alinéa 1 ne soit exigés ${ }^{\circ}$. Le seul refus de la première demande impose au juge de prononcer le divorce pour la deuxième ${ }^{91}$. Dans ce cas, le divorce pour altération est donc de droit, quelle que soit la durée de la séparation92. L’impossibilité de maintenir le lien conjugal est caractérisée, non pas par la séparation, mais par la volonté de chacun des époux de divorcer93. Le fait que les deux époux demandent le divorce, l'un pour faute et l'autre pour altération définitive, fait apparaître l'échec irrémédiable du mariage et l'impossibilité du maintien de l'union ${ }^{94}$.

\section{B. Divorce non sanctionné par les effets}

Si l'ancien divorce pour rupture de la vie commune faisait peser systématiquement toutes les charges et conséquences du divorce sur le demandeur, la loi du 26 mai 2004 a choisi le contraire. Les effets du divorce, y compris celui pour altération définitive du lien conjugal, sont désormais soumis à un droit commun (1). Il en résulte que ce divorce n'est plus un divorce assumé par une contrepartie (2).

\footnotetext{
${ }^{87}$ L’alinéa 2 de l’article 238 du C. civ prévoit : "Nonobstant ces dispositions, le divorce est prononcé pour altération définitive du lien conjugal dans le cas prévu au second alinéa de l'article 246, dès lors que la demande présentée sur ce fondement est formée à titre reconventionnel".

${ }^{88}$ Circ. min. ${ }^{\circ} \mathrm{CIV} / 16 / 04,23$ nov. 2004.

${ }^{89}$ Hauser (J.), "Divorce pour faute et demande reconventionnelle en altération définitive du lien conjugal : de la hiérarchie des causes de divorce et de la durée de la séparation", RTD civ. 2012, p. 100, note sous Cass. 1re civ., 5 janvier 2012.

$9^{\circ}$ Courbe (P.) et Gouttenoire (A.), Droit de la famille, Sirey, Université, Dalloz, 2017, 7e éd., p. 212.

${ }^{91}$ C'est ce que la Cour de cassation a confirmé le 5 janvier 2012 en déclarant qu'“en cas de présentation d'une demande principale en divorce pour faute et d'une demande reconventionnelle en divorce pour altération définitive $d u$ lien conjugal, le rejet de la première emporte le prononcé du divorce sur la seconde". Cass. 1re civ., 5 janv. 2012, n ${ }^{\circ}$ 10-16.359. D, 2012, n 10, Chron. C. Cass, pp. 635s, note Vassallo (B.) ; RJPF, 2012, nº 2, p. 20, Analyse Garé (Th.) ; D, 2012, nº 3, p. 150, Marrocchella (J.) ; Gaz. Pal, 17 mars 2012, pp. 25s, note Casado (A.-L.) ; JPC G, $\mathrm{n}^{\circ}$ 3- 16 janvier 2012, p. 76, note Coutant-Lapalus (C.) ; JPC G, nº 8- 20 février 2012, p. 361, note Massip (J.) ; AJ Fam, 2012, n 2, p. 104, Obs. David (S.) ; RTD civ, 2012, p. 99, note Hauser (J.).

${ }^{2}$ GARÉ (Th.), "Le rejet de la demande principale en divorce pour faute emporte le prononcé du divorce pour altération définitive du lien conjugal", RJPF, 2012, $\mathrm{n}^{\circ}$ 2, p. 20, Analyse de Cass. 1re civ., 5 janv. 2012, $\mathrm{n}^{\circ}$ 10-16.359; Cheynet de Beaupré (A.), "Spécificité du divorce pour altération définitive du lien conjugal après rejet de la demande reconventionnelle en torts exclusifs", RJPF, 2013, $\mathrm{n}^{\circ} 11$, sélection du mois, p. 22.

93 Coutant-Lapalus (C.), "Un divorce pour altération définitive du lien conjugal sans séparation”, JCP G, 16 janvier, 2012, $\mathrm{n}^{\circ} 3$, p. 77.

94 Massip (J.), "Larticle 238, alinéa 2, du Code civil et le domaine du divorce pour altération définitive du lien conjugal", note sous Cass. 1re civ., 5 janv. 2012, $\mathrm{n}^{\circ}$ 10-16.359, JCP G, 2012, n ${ }^{\circ}$ 8, p. 362. En ce sens aussi : Hauser (J.), "Divorce pour faute et demande reconventionnelle en altération définitive du lien conjugal : de la hiérarchie des causes de divorce et de la durée de la séparation", op. cit., p. 100.
} 


\section{Standardisation des effets du divorce sous le droit commun}

Pour banaliser plus encore le divorce, la réforme de 2004 a abrogé les conséquences très lourdes qui pesaient sur le demandeur du divorce pour cause objective. En alignant les conséquences de ce divorce sur celles des autres cas $^{95}$, le législateur français a standardisé les effets du divorce. Le demandeur n'est désormais plus tenu d’en supporter toutes les charges. Tout divorce, quelle qu'en soit la cause, fait cesser le devoir de secours ${ }^{96}$. Le droit commun gouverne l'ensemble des mesures accessoires de ce divorce. La prestation compensatoire ${ }^{97}$ est appréciée pour les deux époux, défendeur comme demandeur du divorce pour altération, selon les conditions posées en droit commun ${ }^{98}$. Il en résulte que l'époux qui a imposé la rupture peut obtenir, éventuellement ${ }^{99}$, une prestation compensatoire dès lors que le divorce qu’il réclame crée une disparité dans les conditions de vie respectives des époux ${ }^{100}$. L'époux le moins fortuné pourra donc réclamer à son conjoint une prestation compensatoire ${ }^{101}$. La philosophie du législateur de la réforme de 2004 a profondément changé en détachant les causes du divorce de ses effets. La rupture par une volonté unilatérale ne donne plus de privilège quelconque au défendeur du divorce. Celui-ci n'est plus considéré comme victime. [...] les deux conjoints sont traités de la même manière ${ }^{102}$. La seule consolation pour l'époux défendeur ${ }^{103}$, dans le domaine des effets du divorce, réside dans la possibilité de demander une réparation sur le fondement de l'article $266 \mathrm{du}$ C. civ $^{104}$. Nonobstant, pour l'obtenir, deux critères doivent être réunis selon cet article :

1. Que le défendeur subisse des conséquences d'une particulière gravité du fait de la dissolution du mariage par le demandeur de la réparation.

${ }^{95}$ À l'exception de la charge des dépenses de l'instance qui, selon l'article 1127 CPC, est devenue facultative. BÉNAbent (A.), Droit de la famille, op. cit., p. 199; Murat-Sempietro (M.-P.) et Trambouze (V.), Le divorce après la loi du 26 mai 2004, collection de l'Institut national de formation notariale, Pratique professionnelle, Lexis Nexis Litec, Paris, 2006, p. 20.

${ }^{96}$ L’alinéa 1 de l'article 270 C. civ prévoit : "Le divorce met fin au devoir de secours entre époux". Alors le devoir de secours est logiquement supprimé. V. Murat-Sempietro (M.-P.) et Trambouze (V.), Le divorce après la loi du 26 mai 2004, op. cit., p. 21.

97 La prestation compensatoire est une somme d'argent forfaitaire que l'un des époux peut se trouver tenu de verser à l'autre après le divorce pour compenser la disparité que la rupture du mariage crée dans les conditions de vie respectives. L'évaluation de cette somme prend en compte : l'âge, l'état de santé des époux, leur patrimoine, etc. Cette prestation, en principe non révisable, peut être fixée sous la forme soit d'un capital, soit d'une rente indexée. Cornu (G.), Vocabulaire juridique, Association Henri Capitant, Puf, 11e éd., 2016. p. 213.

${ }^{98}$ Selon l'alinéa 2 de l'article 270 du C. civ : "Lıun des époux peut être tenu de verser à liautre une prestation destinée à compenser, autant quil est possible, la disparité que la rupture du mariage crée dans les conditions de vie respectives. Cette prestation a un caractère forfaitaire. Elle prend la forme diun capital dont le montant est fixé par le juge".

99 Dans ce cadre, force est d'indiquer que la déchéance exceptionnelle pour équité, prévue dans l'alinéa 3 de l'article $270 \mathrm{du}$ C. civ, ne concerne que le divorce prononcé aux torts exclusifs d'un époux. Cet alinéa prévoit que: "Toutefois, le juge peut refuser diaccorder une telle prestation si liéquité le commande, soit en considération des critères prévus à liarticle 271, soit lorsque le divorce est prononcé aux torts exclusifs de liépoux qui demande le bénéfice de cette prestation, au regard des circonstances particulières de la rupture".

${ }^{100}$ Claux (P.-J.), David (S.) et autres, Droit et pratique du divorce, op. cit., p. 37; Malaurie (Ph.) et Fulchiron (H.), Droit de la famille, L.G.D.J, Collection Droit privé, 5e éd., 2016, p. 330.

${ }^{101}$ Garé (Th.), "Présentation de la réforme", in La réforme du divorce par la loi de 26 mai 2004 : pacification et simplification?, op. cit., p. 19.

102 J. Garrigue, Les devoirs conjugaux "Réflexion sur la consistance du lien matrimonial ", op. cit., p. 374.

${ }^{103}$ Selon l'expression du Doyen H. Fulchiron, "Les métamorphoses des cas de divorce", Rép. Defrénois, 15 septembre 2004, n 17, art. 37999, Doct, p. 1115.

${ }^{104}$ Le défendeur peut aussi demander des dommages et intérêts sur le fondement de l'article $1382 \mathrm{du}$ C. civ. 
2. Qu'il n’ait formé aucune demande de divorce. Rien n’assure, alors, que le défendeur au divorce pour altération obtienne des dommages-intérêts ${ }^{105}$.

Pour conclure, à l'exception des éventualités de dommages-intérêts et de prestation compensatoire, le demandeur du divorce pour altération ne paie aucun prix financier. Sans doute, le changement remarquable à lrégard des effets du divorce pour altération reflète une grande évolution législative à l'égard de ce type de rupture. Autrement dit, la rupture imposée par un époux n'est plus stigmatisée; à présent, [ce] choix est respecté106. La rupture sans grief est devenue un droit pour celui qui le réclame et le droit ne peut être sanctionné par ses effets. Ce type de rupture a cessé dêtre considéré comme une faute qui doit être sanctionnée par des effets pécuniaires lourds et insupportables.

\section{Droit au divorce sans contrepartie assumée}

La suppression significative des conséquences lourdes dans le divorce pour altération définitive du lien conjugal, consacrées par la loi de 1975, reste une source de critique doctrinale. Certains auteurs, en commentant cette suppression, parlent d' une liberté sans responsabilité ${ }^{107}$, d' une répudiation libre, sans contraintes financières ${ }^{108}$ ou du moins d' Un pouvoir, pas de contrepartie ${ }^{109}$. Plus encore, léventualité que l'époux défendeur à ce divorce paie une prestation compensatoire a choqué une partie de la doctrine. Celle-ci considère que le législateur de 2004 a inversé la logique des choses en rendant le conjoint défendeur, qui ne souhaite pas cette rupture, susceptible non seulement de devoir supporter ce divorce mais encore d'avoir à financer la vie nouvelle de son ancien conjoint ${ }^{110}$. Un exemple, plus fréquent qu'on ne le croit selon le Maître Casey, peut illustrer cette situation. Une personne est à cinq ans de la retraite et après trente-cinq ans de mariage, son conjoint qui n’a jamais travaillé veut divorcer. Le conjoint qui ne veut pas le divorce doit nécessairement quitter le domicile conjugal et se reloger puisqu'il a les revenus. Il doit aussi payer un devoir de secours ainsi qu'une prestation compensatoire conséquente. Ce conjoint qui va subir le divorce n'a commis aucune faute et a travaillé toute sa vie en partie pour l'autre

\footnotetext{
${ }^{105}$ Comme les professeurs Malaurie et Fulchiron l'ont commenté : “On en déduit que sil avait osé former une demande reconventionnelle en divorce pour faute, le droit lui serait fermé, à moins quil n>ait obtenu le prononcé d`un divorce aux torts exclusifs, l>article 266 jouant également dans cette hypothèse. L`époux défendeur à un divorce pour altération définitive du lien conjugal est donc placé face à une alternative : subir (en espérant être consolé par l>octroi de quelques dommages-intérêts) ou se battre jusqu>au bout en formant une demande reconventionnelle pour faute afin d>obtenir un divorce aux torts exclusifs lui permettant diéchapper, si le juge en est d>accord, au paiement dıune prestation compensatoire et, éventuellement, d`obtenir des dommages-intérêts fondés sur l'article 266. Le pari est hasardeux car il risque de tout perdre si le divorce est finalement prononcé aux torts partagés". Malaurie (Ph.) et Fulchiron (H.), Droit de la famille, op. cit., p. 331.

${ }^{106}$ Garrigue (J.), Les devoirs conjugaux "Réflexion sur la consistance du lien matrimonial”, op. cit., p. 374.

107 Malaurie (Ph.), “Conclusion sur la réforme du divorce: Le divorce pour altération définitive du lien conjugal et la société de la peur”, Rép. Defrénois, 15 décembre 2004, n²23, art. 38062, p. 1602.

${ }^{108}$ Koumdadji (A.), La sécularisation de la répudiation: ou l'avènement de la rupture unilatérale et discrétionnaire en droit français, op. cit., p. 215.

109 Garrigue (J.), Les devoirs conjugaux "Réflexion sur la consistance du lien matrimonial”, op. cit., p. 384.

${ }^{110}$ Malaurie (Ph.) et Fulchiron (H.), Droit de la famille, op. cit., p. 331. Adde Labbée (X.), Le droit commun du couple, op. cit., p. 184. Les Doyens Malaurie et Fulchiron, de leur côté, estiment, en commentant ce point, que "liéquité (on n>ose dire la morale) aurait voulu que le juge puisse au moins refuser dıaccorder une prestation compensatoire en considération des circonstances de la cause, comme il peut le faire si le divorce est prononcé aux torts exclusifs dıun époux [...]”. Malaurie (Ph.) et Fulchiron (H.), Droit de la famille, op. cit., p. 331.
} 
conjoint qui a le droit de jouir de sa liberté de divorcer ${ }^{111}$. À cet égard, le mariage est devenu... un risque! !12.

En fait, il parait choquant que le demandeur du divorce pour altération, qui a décidé discrétionnairement la rupture du mariage, obtienne cette prestation. Mais dans l'analyse complète de ce divorce, il ne faut pas oublier que le demandeur paie aussi au moins deux ans de sa vie pour seulement engager ce divorce. Chaque époux paie alors et de manière différente. Le législateur français est, bien évidemment, appelé à remettre en cause le divorce pour altération définitive du lien conjugal. Ce type de divorce doit traiter les deux parties d'une manière plus juste en prenant en compte les intérêts des deux époux. L'intérêt du demandeur du divorce d'engager un divorce sans délai quelconque et l'intérêt du défendeur de ne pas prendre financièrement en charge son ex-époux ${ }^{113}$.

\section{Conclusion}

La rupture par la volonté unilatérale d'un époux pour cause objective se caractérise par l'absence de faute imputable au défendeur qui subit ce divorce. L'expérience législative française dans ce type de divorce, d'abord en 1975 puis en 2004, reflète un profond changement de regard et un positionnement radicalement différent. En 1975, on constatait peu de liberté au divorce pour le demandeur et un avantage considérable pour le défendeur. À l'inverse, on relève en 2004 plus de liberté au divorce pour le demandeur et pas d`avantage particulier pour le défendeur. La cause objective que le législateur avait partiellement intégrée dans les cas de divorce en 1975 a connu un progrès considérable en 2004, révélant une réelle évolution législative en matière de divorce par une volonté unilatérale d`un époux sans grief imputable à l autre conjoint. Cette volonté qui était stigmatisée, traitée comme coupable de la rupture du mariage est devenue, aujourd hui, titulaire d`un droit. Un droit au divorce qui nétait pas concevable avant 2004. On est passé d`une culpabilité systématique à un droit au divorce décomplexé pour le demandeur de ce divorce. Dıun époux culpabilisé et pénalisé par les effets du divorce à un époux titulaire d'un droit au divorce sans contrepartie. L’évolution est considérable du fait que ce qui était stigmatisé hier est aujourd'hui bienvenu. Du divorce pour cause objective restreinte au divorce pour cause objective plus libérale, la conception du législateur français moderne a évolué à l'égard du mariage lui-même. Le mariage, aujourd'hui, s`alimente d`un consentement mutuel qui doit être renouvelé chaque jour. Pour en sortir unilatéralement, il n`est plus nécessaire d`exiger la preuve d`une faute conjugale imputable à lıautre époux. Il suffit, seulement, que le demandeur de la rupture ne souhaite plus continuer sa vie avec lrautre conjoint. Le législateur français est, tout de même, appelé à réformer ce type de divorce pour réaliser un équilibre plus modéré entre les intérêts opposés des deux époux. L`intervention espérée de la part du législateur français devrait viser à donner encore davantage de liberté au divorce en supprimant le délai de deux ans et à dispenser le défendeur d`une éventuelle prestation compensatoire pour le demandeur du divorce.

\section{La modération en tout est avantage comme "liexcès en tout est défaut ${ }^{114}$.}

\footnotetext{
${ }^{111}$ CAsey (J.), “Faut-il réformer le divorce ?”, Gaz. Pal, 23 janvier 2010, n² 23, p. 10.

${ }^{112}$ Ibid.

${ }^{113}$ Pour plus de détails sur ce point, v.: Alati (A.), "La rupture du mariage par la volonté unilatérale de liépouse :

Étude de législation française et de législation islamique appliquée en pays arabes”, Volume I, op. cit., p.465.

${ }^{114}$ Proverbe français attribué à Boutet de Monvel.
} 


\section{Bibliographie}

\section{Ouvrages généraux : manuels, Dictionnaires}

Bonnet (V.), Droit de la famille, manuel, Larcier, 5e éd., 2015.

Brazier (M.), Le nouveau droit du divorce, Éditions Apil, Versailles.

Carbonnier (J.), Droit civil « Introduction, les personnes, la famille, l'enfant, le couple », PUF, Paris, 2e éd., 2017, Vol. I.

Connu (G.), La réforme du divorce (étude de la loi du 11 juillet 1975), Les cours de droit, Paris, 1976.

Droit civil la famille, Domat droit privé, Montchrestien, Paris, 9e éd., 2006.

Vocabulaire juridique, Association Henri CAPITANT, PUF, 11e éd., 2016.

Claux (P.-J.), David (S.) et autres, Droit et pratique du divorce, Dalloz Référence, Dalloz, ze éd., 2013, p. 35.

Courbe (P.) et Gouttenoire (A.), Droit de la famille, Sirey, Université, Dalloz, 2017, 7e éd.

Courbe (P.), Le divorce, Dalloz, Connaissance du droit, 4e éd., 2004.

Fornerod (A.), «Religion et famille en droit interne», in Droit français des religions, Dir. Messner (F.), Prélot (P.-H.) et Woehrling (J.-M.), avec la contribution d'Isabelle Riassetto, Traités, Lexis Nexis, Paris, 2013, p. 1016.

Lindon (R.), La nouvelle législation sur le divorce et le recouvrement public des pensions alimentaires (lois du 11 juillet 1975 : Analyse et commentaire), Litec, 1975.

Massip (J.),

La réforme du divorce, (Commentaire des lois nos 75-617 et 75-618 du 11 juillet 1975 et des textes d’application), t. I, Répertoire du notariat Defrénois.

Le nouveau droit du divorce, Defrénois, 2005.

Malaurie (Ph.) et Fulchiron (H.), Droit de la famille, L.G.D.J, Collection Droit privé, 5e éd., 2016.

Murat (P.) (dir.), Droit de la famille, Droit de la famille, Dalloz-action, 7e éd., 2016-2017.

Murat-Sempietro (M.-P.) et Trambouze (V.), Le divorce après la loi du 26 mai 2004, collection de l'Institut national de formation notariale, Pratique professionnelle, Lexis Nexis Litec, Paris, 2006.

Parquet (M.), Droit de la famille, Bréal, 4e éd., 2014.

Rubellin-Devichi (J.), 
"La diminution de l'importance du mariage "L'admission du divorce par volonté unilatérale"», in Mariage et famille en question, Tome 1, Dir. R. Nerson, Éditions du C.N.R.S, 1978.

«Les grandes réformes en cours dans le droit de la famille en France», in Droit comparé des personnes et de la famille, Bruylant, Bruxelles, 1998.

Roy (O.), Droit de la famille, Collection Montesquieu, Archétype 82, Paris, 2014.

\section{Ouvrages spéciaux, thèses}

Alati (A.), «La rupture du mariage par la volonté unilatérale de l'épouse : Étude de législation française et de législation islamique appliquée en pays arabes», Volume I, Éditions universitaires européennes, 2018.

Blessy (J.-C.), Le principe du divorce objectif étudié dans ses rapports avec la faute en droits français et allemand comparés, th. Nice, 1989.

GARrigue (J.), Les devoirs conjugaux «Réflexion sur la consistance du lien matrimonial», Éditions Panthéon-Assas II, Collection Thèses, 2012.

Himmi-Mehrabi (M.), La dédramatisation du divorce dans la législation contemporaine, th. Paris II, 2006, t.1.

Koumdadj (A.), La sécularisation de la répudiation: ou l'avènement de la rupture unilatérale et discrétionnaire en droit français, Les éditions du Cerf P, 2015.

\section{Articles, notes, observations, communications}

CARbonnier (J.), «La question du divorce Mémoire à consulter», D, 1975, Chron XX, p. 117.

CAsey (J.), «Faut-il réformer le divorce ?», Gaz. Pal, 23 janvier 2010, nº 23, p. 10.

Cheynet de Beaupré (A.), «Spécificité du divorce pour altération définitive du lien conjugal après rejet de la demande reconventionnelle en torts exclusifs», RJPF, 2013, $\mathrm{n}^{\circ} 11$, sélection du mois, p. 22.

Coutant-Lapalus (C.), «Un divorce pour altération définitive du lien conjugal sans séparation», JCP G, 16 janvier, 2012, $\mathrm{n}^{\circ} 3, \mathrm{p} .77$.

DAvid (S.),

«La clause d'exceptionnelle dureté demeure exceptionnellement admise», note sous CA Paris, 24e ch. A, 11 juin 2003, RG n 2002/02053, AJ Fam, 2003, pp. 384 s.

«Divorce altération: le point de départ du délai de séparation se prouve par tous moyens», Obs. sous Cass. 1re civ., 25 novembre 2009, nº 08-17.117, AJ Fam, 2010, p. 136.

Delecraz (Y.), «Le projet de réforme du divorce», Rép. Defrénois, 15 mai 2004, n 9, art. 37935, p. 647. 
Fulchiron (H.), «Les métamorphoses des cas de divorce», Rép. Defrénois, 15 septembre 2004, $\mathrm{n}^{\circ} 17$, art. 37999, Doct, p. 1115.

FURKel (F.),

«La faute dans le divorce en droit français et allemand», RIDC, Vol. 34, $\mathrm{n}^{\circ}$ 4, octobre-décembre 1982, pp. 1153s.

«La clause de dureté est-elle un mal nécessaire?», D. 1977, Chro X, p. 83.

GARÉ (Th.),

«Pour un réexamen des règles applicables à la clause de dureté fondée sur les règles religieuses», Analyse pour Cass. 2e civ., 13 mars 2003, $\mathrm{n}^{\circ}$ 01-14.616, RJPF, 2003, $\mathrm{n}^{\circ}$ 6, p. 19.

«Le rejet de la demande principale en divorce pour faute emporte le prononcé du divorce pour altération définitive du lien conjugal», $R J P F, 2012, \mathrm{n}^{\circ}$ 2, p. 20, Analyse de Cass. 1re civ., 5 janv. 2012, $\mathrm{n}^{\circ} 10-16.359$.

Gouttenoire (A.), «Les nouvelles procédures de divorce», Rép. Defénois, 2005, n 7, Doct., art. 38137, p. 558.

Granet-Lambrechts (F.), «Les nouveaux cas de divorce», AJ Fam., 2004, p. 207.

HAuser (J.), «Divorce pour faute et demande reconventionnelle en altération définitive du lien conjugal: de la hiérarchie des causes de divorce et de la durée de la séparation», RTD civ. 2012, p. 100, note sous Cass. 1re civ., 5 janvier 2012.

JACQUET (J.-M.), «Le rôle de la cause dans le nouveau droit français du divorce», RTD civ 1984, pp. 625s.

JunG (B.), «Le divorce remède ou la place faite dans le nouveau droit du divorce en France et en République fédérale d'Allemagne», JCP G, 1979, I, 2940.

LARribau-Terneyre (V.),

La réforme du divorce: premier bilan à mi-parcours, Dr. Fam, 2004, n 3, Chron. 6, p. 5.

«Sur la notion de la communauté de vie des époux», note sous : CA Versailles, 2e ch., 31 mars 2011, n 10-02387. Dr. Fam, 2011, n 6, Comm. 94, pp. 30 .

Malaurie (Ph.), «Conclusion sur la réforme du divorce : Le divorce pour altération définitive du lien conjugal et la société de la peur», Rép. Defrénois, 15 décembre 2004, n²3, art. 38062 , p. 1602.

Massip (J.), «L'article 238, alinéa 2, du Code civil et le domaine du divorce pour altération définitive du lien conjugal», note sous Cass. 1re civ., 5 janv. 2012, $\mathrm{n}^{\circ}$ 10-16.359, JCP G, 2012, $n^{\circ} 8$, p. 362 . 
Monsallier (B.), «Le divorce pour rupture de la vie commune (Loi du 11 juillet 1975)», RTD. civ, 1980, p. 267.

\section{Documents officiels: Rapports, notes explicatives, travaux préparatoires}

Annuaire statistique de la justice, La Documentation française, Paris, édition : 2000, pp. 79s, 2001, pp. 81s, 2005, p. 89.

Circulaire de présentation de la loi relative au divorce, du décret portant réforme de la procédure en matière familiale et du décret fixant les modalités de substitution d'un capital à une rente allouée au titre de la prestation compensatoire. Civ 2004-16 C1/23-11-2004. Bulletin officiel du ministère de la Justice nº 96 (1er octobre - 31 décembre 2004).

Dekeuwer-Défossez (F.), Rénover le droit de la famille (Propositions pour un droit adopté aux réalités et aux aspirations de notre temps), Rapport au Garde des Sceaux, ministre de la Justice, La Documentation Française, 1999, p. 114.

Loi $n^{\circ}$ 14-485 de 27 juillet 1884 Loi qui rétablit le divorce en France. Promulguée au J.O du 29 juillet 1884.http://www.justice.gouv.fr/art_pix/Loi_retablissement_divorce_1884.pdf

Rapport pour la Commission des lois, Sénat n 120, présenté par le Sénateur P. GÉLARD, sur le projet de loi relatif au divorce, annexe au procès-verbal de la session du 17 décembre 2003. http://www.senat.fr/rap/lo3-120/lo3-1201.pdf

\section{Décisions de justices}

Cass. 2e civ., 30 janvier 1980, JCP G, 1981. II, J, 19521.

Cass. 1re civ., 5 janv. 2012, $\mathrm{n}^{\circ} 10-16.359 . \mathrm{D}, 2012, \mathrm{n}^{\circ} 10$, Chron. C. Cass, pp. 635 s. 